\title{
LA CARA OCULTA DE LA TRISTEZA
}

THE DARK SIDE OF THE SADNESS

Resumen

A partir de un análisis crítico del vocablo de moda depresión, tratamos de articular, desde el contexto psicoanalítico, la preocupación de la psiquiatría clásica por la tristeza a través del dolor moral. La pérdida, como explicación del duelo, y la melancolía en la obra de Freud, encuentra en la cobardía moral de Lacan el estatuto ético de la depresión: la claudicación del sujeto en relación a su deseo.

Palabras Claves: Depresión. Malentendido. Dolor moral. Pérdida. Cobardía moral.

\section{Summary}

Starting from a critical analysis of the fashionable word depression, we try to articulate, from in the psychoanalytic context, the worry of the classic psychiatrist about the sadness over the pain moral. The loss, like explanation of the mourning, and the melancholy in the Freud's work, finds in the Lacan's moral cowardice the ethical statute of the depression: the individual's subjection in relation to his wish.

Key words: Depression, Misunderstanding, Moral pain, Loss, Moral cowardice.

«Pero no olvidemos, en interés de nuestro estudio, que tampoco el hombre de hoy se siente feliz en su semejanza con Dios.»(1)

S. FREUD 


\section{INTRODUCCIÓN}

Tanto se menciona la palabra depresión -término que podemos acuñar en la actualidad como la manera moderna de nombrar el malestar humano- que, al final, bien poco se acaba por decir sobre lo que bajo esa palabra subyace. En realidad, el término depresión se ha convertido en una expresión que no dice en verdad nada, ni sobre la estructura clínica de quien afirma estar depre ni sobre su posición en relación a esa queja que aparentemente le impide vivir satisfactoriamente. Podríamos decir que, bajo el uso tan abusivo de la voz depresión, «ese término de moda que engloba a los diversos malestares de la modernidad» (2), se cierra el diálogo entre los interlocutores - paciente y profesional- y se sella definitivamente el malestar bajo una etiqueta médica que, aun cuando en principio tranquiliza a ambas partes y permite que «el paciente pueda exigir una pacificación al médico sobre su mal» (3), impide poder escuchar lo que bajo ese velo que enmarca la tristeza se esconde.

Pero entonces, ¿podríamos decir que no existe la depresión como tal? Ni mucho menos. La depresión, como «un estado producido por una mayor o menor exaltación afectiva y displacentera de carácter triste y angustioso que se acompaña de cierto grado de disminución de la actividad motriz, así como de bradipsiquia, temor indefinido o ideación que puede llegar a ser delirante -de impotencia, de rui- na, de culpabilidad- junto a trastornos cenestésicos que pueden concluir en fenómenos hipocondríacos y en casos graves, ideas de negación y de no existencia, tanto de sí mismo como del mundo circundante» (4), existe, aunque, ese estado afectivo para el psicoanálisis no tiene un estatuto estructural autónomo e independiente, sino que más bien forma parte de una queja, un malestar o un sufrimiento, moderno por antonomasia, que insertado en una estructura -neurótica, perversa, psicótica- viene a hablar «no de un estado del alma, sino simplemente de una falla moral, como se expresaba Dante, incluso Spinoza: un pecado, lo que quiere decir una cobardía moral» (5), según palabras de Lacan. ${ }^{1}$

Definir en este punto el afecto de la tristeza como una cobardía moral ante la posición subjetiva ¿es una crítica al estado depresivo, esto es, un reproche inmerecido a ese sufrimiento donde está implicado, digámoslo ya, un obstáculo al deseo? En absoluto. Esa frase, utilizada a modo de provocación por parte de Lacan, es más bien una llamada al sujeto, un envite o un revulsivo, si se quiere, para tratar de sacarlo de esa posición en la que se haya, "confundido en su malestar con el desfallecimiento imaginario de un goce narcisista» (6), y que, apoyado, sin embargo, por los tiempos que corren, no tiene el paciente ninguna vergüenza en reivindicar.

1. No obstante, es necesario diferenciar, a pesar de todo, la melancolía como entidad clínica de la depresión como estado. La primera quedaría encuadrada dentro del campo de la psicosis, mientras que la segunda podría darse en cualquiera de las estructuras clínicas. En este punto, la diferenciación estructural que aportan los mecanismos de represión, renegación y forclusión sobre las estructuras clínicas, marca las diferencias. 
DEPRESI ÓN: ENTRE LA TRANQUI LI DAD DE MUCHOS Y EL MALENTENDIDO DE TODOS

¿Cómo plantear entonces el interés y la proliferación actual de este concepto, tan en boga como impreciso, que a modo de cajón de sastre es utilizado para definir el malestar moderno? El auge del fenómeno depresivo es, a nuestro modo de ver, el resultado de la crisis y atomización de la clínica clásica y de la medicalización del malestar moderno, junto al ascenso imperante en el saber y en nuestra sociedad de consumo del discurso técnico de la ciencia. Un fruto que se hace preciso interrogar.

En primer lugar, es necesario volver a recordar nuevamente que la proliferación de la depresión, como término conceptual y nosológico, es el efecto concomitante del desmoronamiento de la psicopatología clásica en favor de ese sistema de clasificación estadístico y ateórico que suponen los DSM o los ClE, así como del auge imperante actual del discurso científico y técnico. Veamos.

La quiebra de la psicopatología clásica no sólo conllevó al arrinconamiento y la literal desaparición de la paranoia del panorama de la locura (7), sino también a la fragmentación de la histeria de los manuales de la psiquiatría, que no de la clínica, en tanto que la histeria ha sabido adaptarse bien al discurso de la ciencia. No en vano, y como bien sabemos, la fenomenología camaleónica de la histeria -depresión, angustia y somatizaciones difusas en vez del dramatismo conversivo y disociativo de la histeria de antaño- no es más que el in- tento rebelde por adaptarse, gracias a su plasticidad, a los tiempos modernos; esto es, la histérica trata de plegarse en su pasividad rebelde al discurso amo que comanda en cada época(8). De esta forma, y mientras que la histeria ha ido desapareciendo de la nosología psiquiátrica, el malestar neurótico, en cuanto tal, ha ido tomando ese cariz depresivo que, entremezclado con diversas algias, puede ser presentado a los médicos con el escondido deseo de cuestionar su saber. Es la nueva forma de aparición de la insatisfacción neurótica -depresión y dolor físico- en este mundo moderno dominado por la tecnología médica. De ahí que ciertos autores, como G. Wajeman, insis$\tan$ que en realidad la historia de la histeria se confunde con la de su relación con la medicina» (9).

De esta manera, podríamos decir "que, si el siglo XIX proporcionaba a la histérica la entrada en el templo de la ciencia médica sacándola de entre las endemoniadas y las embrujadas» (10), el siglo XX proporciona a los esquizofrénicos y a los depresivos -amén de toxicómanos, anoréxicas y demás desheredados de la sociedad actual- la entrada en un discurso psiquiátrico técnico que, alejándose de la psicopatología clásica, oferta, bajo el elixir químico, la paz deseada. Así, esa psicopatología, que se mostraba entusiasmada con la vivencia del paciente y que en su vivo interés por dialogar con el enfermo trataba de ubicar las diferentes respuestas del paciente en la aparición inefable de los fenómenos intrusivos, parece ya haber sido abandonada en los mantos de la historia, en esa 
historia anhelada, pero definitivamente perdida. Sin embargo, para este menester era necesario poder anular términos como paranoia, histeria e incluso psicosis, no sólo por el valor que siempre habían tenido y siguen teniendo para el psicoanálisis, sino también para poder hacer ingresar definitivamente el capítulo de la locura clásica en el marco de las enfermedades médicas de carácter mental y, con ello, a la psiquiatría, como especialidad médica, en el marco del discurso de la ciencia técnica. Y aquí se hace necesario subrayar la palabra enfermedad por lo que este concepto implica, esto es, por la ausencia de responsabilidad en el sujeto en aquello que le atormenta, así como por la ausencia de cualquier vestigio de subjetividad en aquel mal que presenta. Si antaño la enfermedad mental era considerada como posesión demoníaca, determinación cosmológica o disturbio de humores, en la actualidad, y bajo el epígrafe de enfermedad mental, se anuncia la presencia de un mal en el cuerpo que, sin embargo, carece de participación subjetiva. De esta forma, si en un principio con la palabra enfermedad se había tratado de no ver en el fenómeno de la locura más que el resorte de una alteración morfológica -anatómica antaño, bioquímica en la actualidad y genética o molecular en el futuro-, con el concepto actual de depresión se pretende ver, incluso en la insatisfacción neurótica (la tristeza no delirante, la angustia sobre el futuro o la propia incapacidad para ser feliz), al igual que sucedía con la melancolía clásica de carácter psicótico, el resultado de una alteración anclada en el cuerpo, librándole así en todo momento al paciente de poder hablar sobre su capacidad de decisión en aquello que le compete; esto es, bajo el concepto de enfermedad se le evita al paciente que pueda formular la pregunta por la causa psíquica, y con ello se le ahorra la pregunta en torno a su deseo.

La desaparición, por tanto, de la histeria de la nomenclatura psiquiátrica, en favor de términos más operativos y ateóricos - la esfera de los trastornos como agrupamientos sintomáticos 0 síndromes, no es sólo un simple ataque al psicoanálisis, sino también un intento de hacer desaparecer, incluso en el margen de lo denominado antiguamente como neurótico, ese matiz de subjetividad y responsabilidad en aquello que compete al individuo, esto es, su responsabilidad en la manera de ser y actuar. Es, en este punto, donde el psicoanálisis afirma que más allá de la enfermedad hay un sujeto que padece y está involucrado en ella. De ahí que, frente a la enfermedad sin sujeto de la Medicina de fin de siglo, el psicoanálisis proponga su ética: «! Hay un sujeto en el enfermo porque hay deseo!». $Y$, precisamente, es a partir de esta perspectiva que debemos entender esa frase de Lacan que resalta la depresión como una cobardía moral: un lema que hace de la depresión una cuestión fundamentalmente ética.

Sin embargo, debemos comprender también que este intento de desubjetivación del malestar que se ha propuesto el saber científico actual no es algo aislado de la propia dimensión socio-cultural en que vivimos, sino que precisamen- 
te está arraigado en las bases de nuestra propia cultura. Es, podríamos decir, un efecto de los tiempos por donde se mueve este hombre moderno que camina vertiginosamente por la vida sin querer detenerse a pensar. Cuando en realidad se dice «que vivimos en la era de la ciencia o que lo científico es lo que vale 0 que hay que construir una verdad en base a una realidad científica», lo que definitivamente estamos marcando - con este lenguaje de la ciencia y su instrumento, la técnica- es la forma adecuada de pensar $y$ de relacionarse con el mundo. $Y$ ha sido precisamente hacia este tren científico e instrumental hacia donde la psiquiatría actual se ha encaminado, en un intento por hacerse definitivamente con un discurso médico eficaz, eficiente y efectivo, según la jerga del momento, que le sirviera de puente de conexión con otras disciplinas científicas. Pero no nos equivoquemos; el discurso de la ciencia y de la técnica, llegado a este punto, es el único que vale y cualquier otro modo de pensamiento está condenado al ostracismo. La técnica se ha convertido en la herramienta veraz de cualquier procedimiento de intercambio y resolución de problemas, y apenas se cree ya en otra cosa que no sea lo objetivo, científicamente hablando. Hasta se tiende en la actualidad, por ejemplo, a que la filosofía desaparezca de los programas de enseñanza en favor de la técnica, y, desde luego, lo objetivo en este caso es la máquina, el instrumento de medición, el fármaco y no ya el sujeto, la palabra, ni la relación médico-paciente. El sujeto, la palabra y la relación médico-paciente, como tales, se anhelan en petit comité 0 en los nostál gicos programas televisados como un ideal perdido, pero en realidad apenas se cree ya en ellos. De ahí ese diálogo de sordos con el que en ocasiones se intercambian los mensajes en los medios de comunicación; mensajes que mayoritariamente buscan simplemente dar rienda suelta al goce de cada cual.

Es por todo ello por lo que podemos decir, finalmente, que existe como una unión y una sintonía eficaz entre este sistema científico -que, mediante el discurso técnico de la ciencia, propugna la masiva medicalización del malestar de los sujetos y la búsqueda de un estatuto de enfermedad para la infelicidad humana-, con esta cultura nuestra que impone y sutura cualquier forma de demanda con objetos de consumo que tratan de pervertir el deseo y facilitar el goce.

El resultado final es la anulación de la subjetividad en el sufrimiento humano y la consecuente irresponsabilidad de todo aquello que bajo la queja e, incluso, el acto se cierne; esto es, se cuestiona en todo momento la propia posición del sujeto; algo frente a lo que el psicoanálisis se rebela, porque, como apunta Lacan, de nuestra posición de sujeto siempre somos responsables.

- LA PSIQUIATRÍA CLÁSICA. UNA MIRADA ALREDEDOR DEL DOLOR MORAL. Para tratar de situar ahora el problema de la depresión en el contexto de la cobardía moral, se hace preciso realizar una incursión previa por el campo de la psiquiatría clásica, en tanto que esta dis- 
ciplina se ha mantenido como un marco de referencia y reflexión para el pensamiento psicoanalítico. No en vano, podríamos decir que el establecimiento de la clínica psiquiátrica clásica tendría como su heredera formal a la clínica psicoanalítica.

Sin ánimo de hacer un recorrido demasiado exhaustivo, sí quisiera mencionar a algunos de los autores que me sirven como introducción en el tema sobre la ética (la posición del sujeto) y la depresión, siguiendo atentamente el esquema de trabajo realizado por Quinet (11).

A mediados del siglo XIX, Joseph Guislain propone el término de frenopatías, en lugar de la palabra locu$r a$, para hablar de las enfermedades mentales, en tanto que entendía que las manifestaciones psíquicas eran reacciones psicológicas a un estado de dolor moral. Esta idea de un dolor inicial de carácter moral, nos dirá Bercherie, "aparecería incluso antes de las locuras de tonalidad afectiva alegre» (12). De esta manera, comenzará a irrumpir en el marco de la psiquiatría lo que vendría a ser la principal característica de la melancolía, la frenalgia -phrenalgie-. Un término que será traducido más tarde por los psiquiatras franceses y alemanes, como dolor moral, y que, posteriormente Lacan nombrará, como "dolor de existir», esto es, como el efecto traumático del Lenguaje sobre el viviente. Esta descripción de la melancolía por parte de Guislain, como un estado de exaltación moral (intensificación de los sentimientos de tristeza y manía), se verá reforzada años más tarde por Griesinger, quien caracterizara tam- bién a la melancolía por el dolor moral. Nos dice: «el doliente está cada vez más dominado por un estado de dolor moral que persiste por sí mismo, y que está aún más reforzado por cada impresión moral exterior» (13). Nuevamente, la cuestión ética sigue pesando, a nuestro modo de ver, en el estatuto de los trastornos afectivos.

Pero, ¿cómo vienen todos estos autores a definir este dolor moral? Como un incierto sentimiento de opresión, ansiedad y tristeza. Parecería que se estuvieran adelantando en su descripción a la nomenclatura de los trastornos mixtos de ansiedad y depresión de la ClE-10, aunque para estos autores, y más allá de la importancia que la fenomenología adquiere en su obra, también está presente el correlato moral, el estatuto ético.

No obstante, y a pesar del esfuerzo realizado por todos estos autores para describir y comprender el acontecer morboso, sus descripciones no encuentran cómo responder a la pregunta por la etiología, quedando pendiente por resolver en todos ellos la cuestión de la causa. Parecería, así, que esa tristeza que embota, aturde e invade al paciente debería tener una causa y, sin embargo, antes de que el paciente pueda preguntarse por ella, la respuesta le adviene en forma de esa cascada de pensamientos lúgubres, presentimientos vagos, temores inciertos y sueños angustiosos. ¿Cómo responder entonces a la pregunta por la causa? Si para Griesinger, como para el resto de los clásicos, en el origen del dolor moral del melancólico se sitúa una causa desconocida, para Freud, como veremos, una pér- 
dida vendrá a ser el detonante de ese dolor moral que corroe y destruye por dentro.

A finales del siglo XIX, Sèglas, un psiquiatra francés, viene a dibujar finamente el cuadro de la melancolía simple o hipocondría moral. Un cuadro clínico donde el dolor moral y las alteraciones cenestésicas e intelectivas definen claramente ese estado de melancolía con conciencia. En este estado melancólico, la aparición de las ideas delirantes de ruina, culpabilidad o persecución no son más que la consecuencia de ese dolor moral previo que atormenta al paciente. De esta forma, y aun cuando la temática delirante en el sujeto melancólico pueda ser variada, se presenta sin embargo, y como denominador común, el dolor moral y las alteraciones corporales e ideativas. Nuevamente podemos volver a percibir cómo, en su reflexión, Sèglas detecta en el origen del cuadro una falta moral, precisamente en el mismo lugar en donde Freud evidenciará más tarde la existencia de una pérdida desconocida, esto es, una pérdida de carácter inconsciente.

Si precisamente la falta moral en su origen justifica la presencia de las alteraciones corporales, así como las alteraciones intelectivas, el delirio viene a ser la reacción a ese dolor moral en un intento por comprender intelectivamente la causa del sufrimiento. Vemos, así, cómo el delirio cumple una función de explicación, que, en ocasiones y de forma retroactiva, da contenido a esa causa desconocida que, mediante lo acontecido o no acontecido en la historia previa del suje- to, es el origen del sufrimiento melancólico actual. De ahí esa resignación tan constante con que el sujeto melancólico espera su castigo; un castigo que en ocasiones no duda él mismo en ejecutarlo.

Finalmente, y llegado a su punto extremo, el sujeto melancólico puede llegar a negar su propia vivencia corporal e, incluso, su existencia como ser humano (delirio de negación de Cotard). La muerte del sujeto, como dirá Lacan, antecede en este caso a esa muerte corporal imposible que jadea y anhela el sujeto melancólico.

\section{Y EN ESTO LLEGÓ FREUD. LA TRISTE-} ZA Y LA PÉRDIDA.

La visión freudiana trató de dotar a la psiquiatría clásica, marcada por la descripción de los fenómenos, de un instrumento de comprensión necesario que permitiera aclarar aquella parte que subyace en el fenómeno de la tristeza, esto es, trató de comprender la dinámica de la estructura. El texto de Freud Duelo y melancolía (1915) es, precisamente, el intento de poner el acento en la pérdida inconsciente, como causa de ese dolor moral descrito por los psiquiatras clásicos.

Freud, coetáneo de las grandes discusiones clasificatorias de la enfermedad mental durante principios del siglo XX, no se entretuvo en dilucidar fenomenológicamente la cuestión diagnóstica de la melancolía, sino que trató de aportar una descripción sencilla que permitiera abordar rápidamente la dinámica en juego. La melancolía, nos dice, «se caracteriza psíquicamente por un estado de áni- 
mo profundamente doloroso, una cesación del interés por el mundo exterior, la pérdida de la capacidad de amar, la inhibición de todas las funciones y la disminución del amor propio - sentimiento de autoestima-. Esta última se traduce en reproches y acusaciones, de que el paciente se hace objeto a sí mismo, y puede llegar incluso a una delirante espera de castigo» (14). Sin embargo, y aun cuando la analogía del cuadro del duelo con la melancolía - reacción a la pérdida de un ser amado o de una abstracción equivalente: patria, libertad, ideal...- es superponible en muchos casos, un aspecto resulta claramente diferencial entre ambos: la perturbación de la autoestima marca y viene a delimitar claramente el cuadro de la melancolía.

¿Cómo se produce el duelo?, se pregunta Freud. En el duelo, la realidad pone en evidencia la falta del objeto amado y «demanda que la libido abandone todas sus ligaduras» (15), siendo precisamente todo el trabajo del duelo el esfuerzo necesario para conseguir librarse de tan pesada carga y plegarse de esta manera al mandato de la realidad. Si el objeto ya no existe, el yo debería tratar de liberarse de todos los puntos de enlace con el objeto perdido. De esta manera, para Freud, el trabajo del duelo acaba con la aparición de la libertad del yo y la desaparición de toda inhibición.

$\mathrm{Si}$ en el contexto del duelo la causa de la pérdida es notoria - de ahí que nos resulte fácilmente comprensible el duelo-, en el caso de la melancolía a veces no es tan fácil averiguar qué es lo que realmente se ha perdido 0 , incluso, lo que con el objeto perdido se ha llegado a perder. "De este modo nos veríamos impulsados a relacionar la melancolía con una pérdida de objeto sustraída a la conciencia, diferenciándose así del duelo, en el cual nada de lo que respecta a la pérdida es inconsciente»(16).

Pero no sólo nos llama la atención en la melancolía la naturaleza inconsciente de la pérdida, sino también esa disminución de la autoestima, esto es, ese empobrecimiento del yo, principalmente moral, en donde los reproches, los insultos y la espera de castigo dan cuenta de un ser despreciable -un objeto abyecto (objeto a) - y abandonado del amparo del Otro, como dirá Lacan. Un ser que no muestra, en ningún momento, reparo ni remordimiento alguno en mostrar su falta moral. Freud en este punto no cuestiona tales acusaciones del paciente contra su yo, sino que precisamente su pregunta se basa en «por qué ha tenido que enfermar el sujeto para descubrir tales verdades»(17). Si bien el paciente debe tener buenas razones para haber perdido su autoestima, la pérdida del objeto en la melancolía, a diferencia del duelo normal, ha tenido - culmina diciendo Freud«importantes efectos en el propio yo»(18).

Pero ¿cómo explicar ese autorreproche que ciega severamente la conciencia del melancólico? La conciencia moral, que viene a ser el prolegómeno de la instancia superyoica del modelo estructural freudiano, se separa del yo y dirige sus críticas contra él, como si de un objeto se tratara. Es como si el yo se hubiera convertido en otra cosa bien distinta de lo que previamente era. Pero ¿en qué se 
ha convertido el yo en realidad? En otra persona, en otro objeto; precisamente, en el objeto erótico deseado. Freud nos dirá: «los reproches con los que el enfermo se abruma corresponden en realidad a otra persona, a un objeto erótico, y han sido vueltos contra el propio yo»(19).

Podemos reproducir ahora claramente el proceso de la melancolía. Sobre una elección objetal previa de carácter narcisista, una ofensa real 0 un desengaño convoca la sustracción de la libido hacia otro objeto; pero, en vez de buscar esta libido nuevas fuentes de gratificación en otro objeto, la libido es desplazada directamente al yo, identificándose éste con el objeto abandonado. "La sombra del objeto cae sobre el yo»(20), y con ello el conflicto previo entre el yo y el objeto se transforma «en una disociación entre la actividad crítica del yo y el yo modificado por la identificación»(21). De esta manera, y aun cuando la melancolía tome ciertos aspectos y características del duelo la pérdida real de un objeto erótico, por ejemplo-, ésta mantiene, sin embargo, un mecanismo que le hace específica, esto es, el proceso de regresión de la elección de objeto narcisista al narcisismo, así como ese carácter de sufrimiento placentero (goce) con que el melancólico se atormenta, fruto, por un lado, de la identificación del yo con el objeto perdido y, por el otro, de la satisfacción de las tendencias sádicas dirigidas hacia él. De ahí la facilidad con que puede darse un pasaje al acto -el suicidio-, en tanto que ese yo disociado puede liberar toda su agresividad sobre esa parte del yo identificado con el objeto amado-odiado.
Al final, tanto la melancolía como el duelo, nos dirá Freud, «desaparecen al cabo de un cierto tiempo, sin dejar tras de sí grandes modificaciones»(22). El yo consigue tras el trabajo del duelo desprenderse aparentemente de la libido que se mantenía fijada en el objeto, y acaba, siguiendo las ordenes de la realidad, por alcanzar su libertad anhelada sin triunfo sobre el objeto ni felicidad aparente.

\section{LACAN: LA TRISTEZA, LA COBARDÍA} MORAL Y EL DUELO I MPOSIBLE

Si bien Freud distingue claramente en sus escritos metapsicológicos el duelo y la melancolía -mediante ese mecanismo básico que supone la identificación narcisista al objeto perdido y que hace, por este motivo, que la queja del melancólico se convierta, en realidad, en la fuente de una acusación dirigida hacia al otro-, años más tarde, y a través del desarrollo del modelo estructural -yo, ello y superyo-, Freud convertirá definitivamente a la identificación en el mecanismo básico de construcción del yo, y especialmente del superyo, como heredero de la disolución del complejo de Edipo. De ahí que Lacan, a la hora de hablar de la estructura del duelo en Freud, haga mención precisamente a esa identificación con el objeto perdido, como el elemento esencial del duelo freudiano.

Pero es que, además de esta aportación de Freud al estudio del duelo, mediante el mecanismo de la identificación, podemos decir también que, por esta época, Freud mantiene una interpretación del duelo que resulta por momentos muy 
esperanzadora para todos. Comienza diciendo: «al final de la labor del duelo vuelve a quedar el yo libre y exento de toda inhibición» (23); y, más tarde, para explicar en la melancolía la liberalización del yo frente al objeto, dirá: «el yo puede gozar quizá de la satisfacción de reconocerse como el mejor de los dos, como superior al objeto»(24).

¿Será por tanto posible elaborar radicalmente el duelo 0 , tal vez, ante cualquier duelo se reabre de una manera desgarradora ese duelo imposible de elaborar?

Siguiendo a Freud, la interpretación que hará Lacan de la depresión estará, tanto en el eje de la posición ética del sujeto, en cuanto que la cara oculta de la tristeza es una falla moral o una cobardía moral, como en el eje de su relación con el goce, esto es, con esa satisfacción que atenta contra la vida.

Ya dijimos anteriormente que la introducción de la posición ética en ese mal moderno por excelencia denominado depresión, es la manera provocativa que tiene Lacan de introducir al sujeto en aquello de lo que se queja para hacerle precisamente responsable de aquello que le hace sufrir. Pero, en este punto, conviene aclarar que responsabilidad no es culpabilidad; precisamente, a mayor dosis de culpabilidad menor injerencia de la responsabilidad en el sujeto. No obstante, es cierto que el sujeto depresivo en ocasiones se siente culpable, tanto por Io realizado como por lo no realizado a lo largo de su vida, pero con ello, lo que no quiere escuchar es, precisamente, la parte de responsabilidad en lo acontecido.
Su manera de desresponsabilizarse es culpabilizándose mediante el velo de 10 imaginario. $Y$, en este punto, que duda cabe que la posición del psicoanálisis frente a la psiquiatría del momento es diametralmente opuesta. La psiquiatría, amparándose en la biología, busca desresponsabilizar al sujeto en favor de un déficit de neurotransmisores, por ejemplo, esto es, busca hacerle un enfermo mudo y sordo ante la pregunta sobre la causa, mientras que el psicoanálisis, en tanto que defiende que el hombre no es sólo un organismo sino un cuerpo sometido al efecto del lenguaje, busca la implicación del sujeto en aquello que le hace sufrir, pretendiendo, ante todo, interrogar la relación del sujeto con su goce. Pero el psicoanálisis con ello no trata de moralizar la conducta humana, ni mucho menos de establecer pautas de conducta, sino que favorece a través del rescate de la posición subjetiva en la palabra, aquello que dificulta la puesta en escena del deseo, aquello que le obstruye y le impide surgir. En este sentido, podemos decir que no hay más ética en psicoanálisis que la del deseo, y que la depresión, en este caso, no es más que una cobardía moral frente al deseo, frente a ese deseo inconsciente que nos posee y nos domina.

La tristeza, por tanto, como punto de claudicación frente al deseo, no es más que la cara desfalleciente de ese sujeto que retrocede frente a lo que le determina y que muestra, bajo ese acto de renuncia ${ }^{2}$, una toma de posición pasiva frente a su conflicto, y una reclusión frente a las consecuencias de su deseo; es 
decir el sujeto, refugiándose por momentos -y con el precio tan severo a pagar por ello- en el afecto de la tristeza, se evade de lo que en realidad la produce y retrocede, así, frente al deseo. De ahí que podamos decir que, en realidad, la tristeza esconde, bajo su máscara incierta, la renuncia a saber sobre el inconsciente, esto es, esconde, y evita a la vez, poder confrontarse con la causa de su deseo.

No retroceder frente al deseo se convierte así, en el lema ético del psicoanálisis por excelencia. Pero, ¿a qué deseo nos referimos en realidad? Al deseo inconsciente. $Y$ el deseo inconsciente, como motor del aparato psíquico, no es, desde luego, aquello que desde la consciencia nos va dirigiendo por entre los resortes del consumo, ni siquiera lo que desde la necesidad siempre cambiante se tipifica y encubre bajo la demanda; el deseo, bajo el epígrafe de lo inconsciente, es, en realidad, Io no sabido, lo que nos determina, lo que no se deja cubrir bajo la demanda, lo que no se puede calmar mediante ese objeto que tapa la necesidad, tal y como muy bien nos lo muestra nuestro sujeto anoréxico (25). En definitiva, lo que perdura más allá de la pacificación de la necesidad. El deseo inconsciente por tanto, como efecto del lenguaje sobre el ser vivo, «es la consecuencia en el ser hablante de la castración 0 , lo que es lo mismo, de la diferencia introducida por el significante» (26). Un deseo que, en realidad, apunta a un vacío, a la nada, y que, a la vez que marca con su huella enigmática nuestros actos y decisiones, inquieta, domina y posee al ser humano. De ahí que la relación del sujeto humano frente a su deseo siempre se muestre en el terreno de lo conflictivo, de la duda, de la angustia y que, precisamente, frente a ese terreno incierto que supone el deseo -que permitiría un despertar a la vida-, es frente a lo que el sujeto depresivo claudica, dirime y escapa, mediante un síntoma, la tristeza como afecto, que es, a la vez, tanto la expresión de la claudicación frente al deseo, como la satisfacción de esa parte del sujeto que se opone a él (el goce).

Si hemos dicho que la tristeza es una acto de responsabilidad inconsciente, de voluntad involuntaria (27), que obstaculiza el deseo, ese deseo que en realidad apunta a un vacío, a la nada ¿cómo poder afrontarlo sin llegar a asustarnos? ¿Cómo situarnos frente a esa nada que nos invade y que demuestra nuestra frágil existencia? ¿Cómo poder hacer ese duelo por la nada que somos a pesar de la mascarada del yo? Es evidente que con valentía. Con esa valentía que tiene que ver con poder llegar a situarse más allá del sentido del significante, de la palabra. Porque, si el significante traduce un sentido, sabemos sin embargo, que no todo puede ser dicho con la palabra, que hay un punto en que el significante falla y la palabra queda a la deriva sin poder cernir el significado último. Y este significante inexistente, este no-sentido,

2. La tristeza vendría a ser, por tanto, como un acto de voluntad inconsciente que equivale a no afrontar el deseo. 
este vacío estructural con que se topa el ser humano en su peregrinar por la existencia, nos enfrenta también con una tristeza; pero con una tristeza que no es la del melancólico, que, invadido por un vacío, se deja llevar hacia el goce mortífero de un síntoma de queja que no cesa 0 , en ocasiones, hacia el hilo de una muerte con la que trata de tapar su malestar incierto, sino que es una tristeza, efecto de un duelo imposible de resolver, que confirma que el sentido de la vida es el de un deseo, dirigido hacia la muerte que puede ser trasladado a la vida, aun a sabiendas de lo perecedera que ésta resulta. En fin, una tristeza que no martiriza ya al cuerpo, que no culpabiliza a nadie y que, sin embargo, permite seguir deseando.

De esta forma, si la tristeza de la depresión es una obstrucción al deseo, la tristeza, efecto del duelo imposible pero aceptado sin resignación-, es una invitación a vivir con un deseo siempre vivo y que no se agota. Un deseo que, aun cuando guarda relación con la falta que agujerea al ser parlante, no impide, sin embargo, la realización de una vida que se dirige inevitablemente hacia la muerte.

\section{BIBLIOGRAFÍA}

1. Freud, S., El malestar en la cultura, Madrid, Biblioteca Nueva, 1973.

2. López Herrero, L. S., «El velo de la depre», Revista Horus, 1998, 1, pp 38-39.

3. Rodríguez Eiras, J., "Sobre la clínica de la depresión», Notas freudianas, 1996, 2, pp 63-67.

4. Sobre-Casas, L. F. y otros, La depresión y el reverso de la psiquiatría, Buenos Aires, Paidós, 1997.

5. Lacan, J, Psicoanálisis. Radiofonía \& Televisión, Barcelona, Anagrama, 1977.

6. Pereña, $F$., «No es raro así que tantos se mueran de tristeza», Notas freudianas, 1996, 2, pp 68-71.

7. Alvarez Martínez, J. M., «¿Qué fue de la Paranoia?», La salud mental en los noventa, 1997, IV J ornadas de la Asociación Castellano Leonesa de Salud mental, pp 43-79.

8. Maleval, J.-C., Cómo desembarazarse de la histeria o la histeria en el siglo XX, Rev. Asoc. Esp. Neuropsiq., 1994 XIV (49), pp 269-290.

9. Wajeman, G., Le maitre et I'histerique, París, Navarin, 1982.

10. Angosto Saura, T.; Cibeira Badia, M… A., «La psiquiatría y la histeria hoy», La salud mental en los noventa, 1997, IV J ornadas de la Asociación Castellano Leonesa de Salud mental, pp 91-112.

11. Quinet, A., y otros, La depresión y el reverso de la psiquiatría, Buenos Aires, Paidós, 1997. 
12. Bercherie, P., Los fundamentos de la clínica, Buenos Aires, Manantial, 1986.

13. Quinet, A., y otros, Op. cit. (11)

14. Freud, S., Duelo y Melancolía, Madrid, Biblioteca Nueva, 1973.

15. I bid, pp 2092.

16. I bid, pp 2092.

17. I bid, pp 2093.

18. I bid, pp 2094.

19. I bid, pp 2094.

20. I bid, pp 2095.

21. I bid, pp 2095.

22. I bid, pp 2097.

23. I bid, pp 2092.

24. I bid, pp 2100.

25. López Herrero, L.S., Anorexia: Comer nada. Rev. Asoc. Esp. Neuropsiq.,1999, XIX, 72, pp. 599-608.

26. Coccoz, V., y otros., Variantes de la depresión en las mujeres, Madrid, DGM, 1998.

27. Colina, F., «Tristeza voluntaria e involuntaria», La salud mental en los noventa, 1997, IV J ornadas de la Asociación Castellano Leonesa de Salud mental, pp 81-90.

Luis-Salvador López Herrero

Médico Especialista Medicina Familiar y Comunitaria. Psicoanalista.

Tutor de la Unidad Docente de Medicina Familiar y Comunitaria de León.

Centro de Salud J osé Aguado. León

Cl. Páramo № 1 -5A. Esc. 3. León 24006

Telf. 987-253856

luis_salvador@ terra.es

Ana Pérez

Médico Residente. Medicina Familiar y Comunitaria

Centro de Salud J osé Aguado. León 